\title{
Magnetic Monopole Dark Matter
}

\author{
Christopher B. Verhaaren \\ Department of Physics and Astronomy, University of California, Irvine, California 92697, USA
}

\begin{abstract}
Magnetic monopoles are predicted by many quantum field theories. Non-minimal dark sectors may well include such particles. I show how dark magnetic monopoles can have small, perturbative, couplings to the visible sector, and how they might make up a portion of the observed dark matter. I clarify how such dark monopoles can be detected experimentally, including novel effects in Aharonov-Bohm phase detectors.
\end{abstract}

Keywords: dark matter, magnetic monopoles

DOI: 10.31526/ACP.NDM-2020.14

\section{INTRODUCTION}

Beginning in the last century [1], the evidence for a new dark matter (DM) in the Universe has grown to ever more convincing levels [2]. We now understand that the DM makes up $80 \%$ of the matter in the observable Universe. Understandably, the first hypotheses for identity of the DM came from within the standard model (SM). The observation that DM with SM couplings and mass scale set by the weak nuclear force provides a thermal relic of the observed mass density opened the WIMP paradigm, wherein the DM is often seen as a simple addition to the SM or a motivated SM extension.

While these possibilities are certainly interesting and should continue to be explored, the dark sector may be richer and more diverse. In such scenarios the DM may be made up of many particle species of various masses or a smaller set of particles which are part of a more sophisticated framework. Such viewpoints are motivated by such ideas as Neutral Naturalness [3] and Dynamical Dark Matter [4], and are also well worth exploring. In particular, the dark sector, like the SM sector, can includes gauge symmetries which may or may not be broken.

If a dark non-Abelian gauge symmetry is broken down to a residual $U(1)_{D}$ group, there can be magnetic monopoles in the dark sector [5]. Depending on how the two sectors interact, a kinetic mixing [6] between the dark abelian gauge symmetry and the visible sector E\&M can be generated. As has been pointed out, this leads to interactions between the dark monopoles and SM particles [7, 8, 9]. Since the dark monopoles could make up some fraction of the DM [10,11], the magnetic nature of these particles may be exploited in DM searches. In these proceedings I outline the form and nature of couplings between dark monopole dark matter and the visible photon. I then consider a particular model in which Aharonov-Bohm [12] phase shifts are a leading, and novel, signal of the DM [11].

\section{KINETIC MIXING WITH ELECTRIC AND MAGNETIC CURRENTS}

Including both electric and magnetic charges in quantum field theory is nontrivial. Rather than using nonlocal formulations [13, 14], we employ the manifestly local Lagrangian of Zwanziger [15]:

$$
\mathcal{L}=-\frac{1}{4 e^{2}}\left(F^{\mu v} F_{\mu \nu}^{A}+{ }^{*} F^{\mu v} F_{\mu \nu}^{B}\right)-A_{\mu} J^{\mu}-\frac{4 \pi}{e^{2}} B_{\mu} K^{\mu}
$$

where for a vector field $X^{\mu}$ we use the notation $F_{\mu \nu}^{X}=\partial_{\mu} X_{v}-\partial_{\nu} X_{\mu}$ and

$$
F_{\mu v}=\frac{n^{\alpha}}{n^{2}}\left(n_{\mu} F_{\alpha v}^{A}-n_{v} F_{\alpha \mu}^{A}-\varepsilon_{\mu \nu \alpha \beta} n_{\gamma} F^{B \gamma \beta}\right)
$$

Of course ${ }^{*} F^{\mu v}=\frac{1}{2} \varepsilon^{\mu v \alpha \beta} F_{\alpha \beta}$ as usual. The Euler-Lagrange equations for the $A_{\mu}$ and $B_{\mu}$ vector fields yields

$$
\partial_{v} F^{\mu v}=e^{2} J^{\mu}, \quad \partial_{v}^{*} F^{\mu v}=4 \pi K^{\mu},
$$

which are exactly the Maxwell equations for electric currents $J^{\mu}$ and magnetic currents $K^{\mu}$. So, at the cost of representing the photon by two vector fields $A_{\mu}$ and $B_{\mu}$ we obtain quantum electrodynamics where $A_{\mu}$ couples to electric currents and $B_{\mu}$ couples to magnetic currents.

The four-vector $n^{\mu}$ that appears in Eq. (2) is an arbitrary constant spacelike vector, and is related to the so-called Dirac string. Requiring these strings to have no physical consequences leads to the famous Dirac charge quantization condition

$$
q g=\frac{N}{2},
$$


where $N$ is an integer and $g$ is the magnetic charge in units of $4 \pi / e$. In the Lagrangian, $n^{\mu}$ plays the role of projecting out the extra degrees of freedom on-shell, so that $A_{\mu}$ and $B_{\mu}$ only give rise to two propagating degrees of freedom. However, this means that the action depends on a fixed vector, or in other words, Lorentz invariance is not a manifest symmetry of the Lagrangian. While this is somewhat uncomfortable, we can only require that physical quantities be Lorentz invariant, and it has has been argued [16,17] that this requirement is met.

Within this formalism we can examine kinetic mixing between to $U(1)$ gauge fields, each of which with electric and magnetic couplings. In particular, we outline how the visible photon can obtain a small coupling to dark magnetic monopoles. However, this is only a physical coupling when the dark $U(1)_{D}$ is broken by giving a mass

$$
\frac{1}{2} m_{D} A_{D \mu} A_{D}^{\mu}
$$

to the dark $A_{D}^{\mu}$ gauge field [8,9]. Consequently we focus on this situation. The kinetic mixing term is obtained by considering the loop-level corrections [18] to the kinetic terms, yielding the expected operator

$$
\frac{\epsilon}{2 e e_{D}} F_{\mu \nu} F_{D}^{\mu \nu}
$$

where $e_{D}$ is the gauge coupling of the $U(1)_{D}$ gauge fields. This mixing can be eliminated, to leading order in $\epsilon$, by making the following transformation

$$
\left(\begin{array}{c}
A_{\mu} \\
A_{D \mu}
\end{array}\right)=\left(\begin{array}{cc}
1 & \epsilon e / e_{D} \\
0 & 1
\end{array}\right)\left(\begin{array}{c}
\bar{A}_{\mu} \\
\bar{A}_{D \mu}
\end{array}\right), \quad\left(\begin{array}{cc}
B_{\mu} \\
B_{D \mu}
\end{array}\right)=\left(\begin{array}{cc}
1 & 0 \\
-\epsilon e_{D} / e & 1
\end{array}\right)\left(\begin{array}{c}
\bar{B}_{\mu} \\
\bar{B}_{D \mu}
\end{array}\right) .
$$

which also keeps the visible photon massless in the presence of the mass terms in Eq. (5). The lines over fields and currents denotes that they pertain to the basis without kinetic mixing. The currents that couple to these diagonal fields are

$$
\left(\begin{array}{c}
\bar{J}_{\mu} \\
\bar{J}_{D \mu}
\end{array}\right)=\left(\begin{array}{cc}
1 & 0 \\
\epsilon e / e_{D} & 1
\end{array}\right)\left(\begin{array}{c}
J_{\mu} \\
J_{D \mu}
\end{array}\right),\left(\begin{array}{c}
\bar{K}_{\mu} \\
\bar{K}_{D \mu}
\end{array}\right)=\left(\begin{array}{cc}
1 & -\epsilon e_{D} / e \\
0 & 1
\end{array}\right)\left(\begin{array}{c}
K_{\mu} \\
K_{D \mu}
\end{array}\right) .
$$

The $J^{\mu}$ transformation shows that the dark photon picks up an $\epsilon$ sized coupling to visible sector electrically charged particles, this is the expected dark photon result. At the same time, the $K^{\mu}$ transformation shows that the visible photon has an $\epsilon$ sized coupling to the dark magnetic monopoles. Therefore, even if dark magnetic coupling $g_{D} 4 \pi / e_{D}$ is large, the $\epsilon \ll 1$ coupling between the dark monopoles and the visible electric particles can be small.

This perturbative coupling is only physical when the dark $U(1)_{D}$ is broken. When this occurs the magnetic charges are confined, and may be thought of as connected by tubes of magnetic flux with tension that scales like $m_{D}^{2}$ [19]. Thus, we can investigate the effects of visible sector electric charges interacting with the dark magnetic bound states. We also note that this perturbative charge seems to violate the Dirac charge quantization condition in the visible sector. This is, in fact, the case. There is only one charge quantization that exists for the combination of the visible and dark gauge theories. When the dark $U(1)_{D}$ is broken, the charge quantization condition may be violated by terms that go like $m_{D} / E$. This is why the coupling is only physical below the mass of the dark photon. At energies much larger than $m_{D}$ the effects from $U(1)_{D}$ breaking are small, effectively reducing violations of charge quantization like the small perturbative coupling.

Recall that the charge quantization condition effectively ensure that there are no physical Aharonov-Bohm (AB) phases in the interactions between electric and magnetic charges. Thus, a corollary of the perturbative magnetic charge is the existence of measurable phases. Indeed, it is obvious that the tubes of magnetic flux which bind the magnetic charges will give rise to physical $\mathrm{AB}$ phases in the visible sector due to the kinetic mixing.

\section{MAGNETIC DARK MATTER AND AHARONOV-BOHM}

The magnetic charges are bound together, so particle-antiparticle pairs quickly annihilate. A stable population can exist if there are two flavors of monopoles, one with an asymmetry in particles and the other with a compensating asymmetry in antiparticles, which would ensure a global charge neutrality. When this population is bound together the flavor symmetric bound states quickly annihilate away leaving the stable opposite flavor states.

We are interested in investigating how the AB phase signal can be maximized. To this end, we suppose that the monopole mass $M$ is much larger than the dark photon mass $m_{D}$. In this limit the bound state is nonrelativistic and can be approximated by the Hamiltonian

$$
H=\frac{p^{2}}{2 M}+C m_{D}^{2}|r|
$$

where $C$ is a dimensionless parameter of order one, and $r$ is the separation between the monopoles. Since $M H$ depends on a single dimensionfull parameter we can immediately estimate the energies $E$ and separations $L$ of the bound states

$$
E \sim \frac{C^{2 / 3} m_{D}^{4 / 3}}{M^{1 / 3}}, \quad L \sim \frac{1}{\left(C M m_{D}^{2}\right)^{1 / 3}}
$$


For $M \sim \mathrm{keV}$ and $m_{D} \sim \mathrm{eV}$ we find $E \lesssim 1 \mathrm{eV}$ and $L \sim 10 \mathrm{~nm}$.

These bound states can make up a portion of the dark matter, subject to some constraints. As long as the bound states are never in thermal equilibrium with the SM, they can be cold enough that they do not de-excite by SM photon emission at late times. This requires that the kinetic mixing be $\epsilon \lesssim 10^{-12}$ for the dark photon mass we are investigating. Then there are the usual dark photon bounds [20] for light dark photons. There are also new bounds related to the magnetic nature of the monopoles, but these tend to be subleading, see [11] for a thorough discussion. Finally, galaxy cluster analyses [21] show that DM self-interactions satisfy $\sigma /(2 M) \lesssim 0.47 \mathrm{~cm}^{2} / \mathrm{g}=(13 \mathrm{GeV})^{3}$. If we reason by analogy with atomic scattering, we expect our large magnetic dipoles will exceed these values. However, if these states make up only $10 \%$ or less of the DM these bounds do not apply [22].

We can now estimate the size of the $\mathrm{AB}$ phase shifts that these dark magnetic bound states would generate. We note that in order to measure anything the bound states must have a nonzero expectation of the distance $L$ between the two charges. For instance, in the $S$-wave ground state there is no net direction for the flux, and hence no AB phase. However, the sun, or terrestrial experiments can excite some fraction of the bound states into low lying excited states with $L \neq 0$. Then, the typical definition of the $\mathrm{AB}$ phase leads to

$$
\Phi_{\mathrm{AB}}=e q \oint d \vec{x} \cdot \vec{A} \approx 2 \pi q \frac{e}{e_{D}} g_{D} \epsilon \frac{L}{\sqrt{R^{2}+L^{2}}},
$$

where $q$ is the electric charge of the visible particle, $L$ is the length of the bound state, and $R$ is the characteristic separation between the slits of the $\mathrm{AB}$ set up, essentially a two slit experiment with electrons. We need $R$ to be larger than $m_{D}^{-1}$ in order for a measurable phase to result. This can give a lower limit to the dark photon masses such experiments are sensitive to. By using the existing experimental set-ups with $R \sim \mu \mathrm{m}$ we find that the expected phase shifts are on the order of $10^{-15}$, which is many orders of magnitude smaller than the sensitivities quoted in the mid 1980s $\sim 10^{-} 3$, when the AB effect was confirmed [23]. Clearly, in the last 35 years there have been great improvements in the experimental techniques and technologies. So, while searches of dark matter with the $\mathrm{AB}$ effect would have to be extremely precise, this novel method of searching for dark matter is an exciting prospect.

In summary, the possibility of rich dark sectors should be explored. Such sectors can contain magnetic monopoles. We have shown that in such cases there can be physical, but perturbative, couplings between the visible photon and the dark monopoles when there is kinetic mixing and the dark $U(1)_{D}$ is broken. These interactions may give rise to novel effects, and in particular could lead to dark matter being discovered through sensitive measurements of Aharonov-Bohm phases.

\section{ACKNOWLEDGEMENTS}

This article describes work done in collaboration with John Terning. The work of C.B.V is supported in part by NSF Grant No. PHY-1915005 and in part by Simons Investigator Award \#376204.

\section{References}

[1] V. C. Rubin, W. K. Ford, and N. Thonnard, Astrophys. J. 225 (1978) L107.

[2] D. Clowe et. al., Astrophys. J. 648 (2006) L109.

[3] Z. Chacko, H. Goh and R. Harnik, Phys. Rev. Lett. 96 (2006), 231802

[4] K. R. Dienes and B. Thomas, Phys. Rev. D 85 (2012), 083523

[5] G. 't Hooft, Nucl. Phys. B 79 (1974) 276; A. M. Polyakov, JETP Lett. 20 (1974) 194 [Pisma Zh. Eksp. Teor. Fiz. 20 (1974) 430].

[6] B. Holdom, Phys. Lett. 166B (1986) 196.

[7] F. Brummer and J. Jaeckel, Phys. Lett. B 675 (2009) 360; F. Brummer, J. Jaeckel and V. V. Khoze, JHEP 0906 (2009) 037.

[8] A. Hook and J. Huang, Phys. Rev. D 96 (2017) 055010.

[9] J. Terning and C. B. Verhaaren, JHEP 12 (2018), 123

[10] C. Gomez Sanchez and B. Holdom, Phys. Rev. D 83 (2011) 123524; S. Baek, P. Ko and W. I. Park, JCAP 1410 (2014) 067; V. V. Khoze and G. Ro, JHEP 1410 (2014) 061; V. V. Burdyuzha, J. Exper. and Theor. Phys. (2018) 127638.

[11] J. Terning and C. B. Verhaaren, JHEP 12 (2019), 152

[12] Y. Aharonov and D. Bohm, Phys. Rev. 115 (1959) 485.

[13] P. A. M. Dirac, Proc. R. Soc. A 133 (1931) 60; Phys. Rev. 74 (1948) 817.

[14] J. S. Schwinger, Phys. Rev. 144 (1966) 1087; Phys. Rev. 173, (1968) 1536; Phys. Rev. D 12 (1975) 3105.

[15] D. Zwanziger, Phys. Rev. D 3 (1971) 880

[16] R. A. Brandt, F. Neri and D. Zwanziger, Phys. Rev. Lett. 40 (1978), 147-150; Phys. Rev. D 19 (1979), 1153

[17] J. Terning and C. B. Verhaaren, JHEP 03 (2019), 177

[18] L. V. Laperashvili and H. B. Nielsen, Mod. Phys. Lett. A 14, 2797 (1999)

[19] H. B. Nielsen and P. Olesen, Nucl. Phys. B 61 (1973) 45.

[20] R. Essig et al, arXiv:1311.0029 [hep-ph].

[21] D. Harvey, R. Massey, T. Kitching, A. Taylor and E. Tittley, Science 347 (2015), 1462-1465

[22] J. Fan, A. Katz, L. Randall and M. Reece, Phys. Dark Univ. 2 (2013), 139-156; Phys. Rev. Lett. 110 (2013) no.21, 211302

[23] R. G. Chambers, Phys. Rev. Lett. 5 (1960) 3; A. Tonomura, et. al. Phys. Rev. Lett. 56 (1986) 792. 